\title{
Efeito de um Programa de Treinamento da Memória de Trabalho em Adultos Idosos
}

\author{
Effect of a Working Memory Training Program in Older Adults
}

\author{
Tânia Maria Netto ${ }^{a, b}$, Denise Vieira Greca ${ }^{a, b}$, Nicolle Zimmermann ${ }^{c}$, Camila Rosa de Oliveira ${ }^{c}$, \\ Homero Marinho Teixeira-Leite ${ }^{d}$, Rochele Paz Fonseca ${ }^{c} \&$ J. Landeira-Fernandez ${ }^{*}$,,$e$ \\ ${ }^{a}$ Pontifícia Universidade Católica do Rio de Janeiro, Rio de Janeiro, Brasil, \\ ${ }^{b}$ Universidade Federal do Rio de Janeiro, Rio de Janeiro, Brasil, \\ ${ }^{c}$ Pontifícia Universidade Católica do Rio Grande do Sul, Porto Alegre, Brasil, \\ ${ }^{d}$ Universidade Estadual do Rio de Janeiro, Rio de Janeiro, Brasil \\ \& ${ }^{e}$ Universidade Estácio de Sá, Rio de Janeiro, Brasil
}

\begin{abstract}
Resumo
Este estudo objetivou examinar os efeitos de um Treinamento da Memória de Trabalho (TMT) em idosos saudáveis. Vinte participantes compuseram a amostra final, onze do grupo experimental (TMT) e nove do controle (socialização). Todos foram submetidos a uma avaliação neuropsicológica pré e pós-intervenção. Os encontros foram realizados uma vez por semana, durante três meses. Houve melhora significativa, no grupo experimental, em atenção concentrada, aprendizagem, memória de curto prazo e episódica, e no grupo controle, em um menor número de variáveis, na atenção concentrada e memória episódica. O TMT parece ter promovido efeito de transferência, principalmente na memória episódica, que é relacionada diretamente aos subsistemas da memória de trabalho (MT), sugerindo que o TMT pode ser útil no contexto da neuropsicologia do envelhecimento.

Palavras-chave: Memória de trabalho, memória episódica, treinamento neuropsicológico, idoso.

Abstract

The present study examined the effects of a Working Memory Training (WMT) in healthy elderly. Twenty participants comprised the final sample, eleven from the experimental group (WMT) and nine from the control group (socialization). Every subject underwent a neuropsychological evaluation pre and postintervention. The meetings were held once a week, for three months. Results indicated that subjects in the experimental group improved cognitive functions related to concentrated attention, learning, short-term and episodic memory. Subjects in the control group also demonstrated, in a smaller number of variables, improvement in concentrated attention and episodic memory. WMT seems to have generated a transfer effect, especially to episodic memory, which is directly related to the subsystem of working memory (WM), suggesting that WMT may be useful in the context of the neuropsychology of aging.

Keywords: Working memory, episodic memory, neuropsychological training, older adults.
\end{abstract}

Dentre as funções cognitivas estudadas pela neuropsicologia, a memória de trabalho (MT) vêm se destacando por sua complexidade e alta relação com demais processos neuropsicológicos (Baddeley, 2010; Baddeley, Allen, \& Vargha-Khadem, 2010; Cabeza et al., 2004; Leigh \& Hirsch, 2011; Levens, \& Gotlib, 2010). Esse domínio cognitivo constitui-se em um sistema ativo, composto por múltiplos componentes, que possuem a capacidade de armazenar informações por um tempo limitado (de alguns segundos a um ou dois minutos), mas sufi-

\footnotetext{
${ }^{*}$ Endereço para correspondência: Rua Marques de São Vicente, 225, Departamento de Psicologia, Pontifícia Universidade Católica do Rio de Janeiro, Gávea, Rio de Janeiro, RJ, Brasil 22451-041. E-mail: landeira@puc-rio.br
}

ciente para manipular uma ampla variedade de informações durante o processamento de tarefas cognitivas complexas (Baddeley, 2003).

A MT é composta pelo sistema executivo central e três subsistemas auxiliares: alça fonológica, esboço visuoespacial e retentor episódico, sendo que esse último foi adicionado recentemente (Baddeley, 2000; Baddeley \& Hitch, 1974). O executivo central é considerado o subsistema mais importante da MT, pois controla a atenção, coordena, manipula e modifica informações de diferentes origens e seleciona estratégias cognitivas. De acordo com estudos recentes, o executivo central parece ter duas formas de controle atencional para resolver conflitos, uma é automática, baseada nos hábitos aprendidos e a outra, é o Sistema Supervisor Atencional (Supervisory Attentional System [SAS]). Esse último é ativado quando resoluções 
de conflitos, baseadas no controle automático, não são possíveis, como em situações novas (Baddeley, 2009a; Norman, \& Shallice, 1986; Oliveira, 2007).

Dos sistemas considerados auxiliares, a alça fonológica armazena informações verbais em um processo de recapitulação articulatória por curto prazo, tempo suficiente para que tais informações possam ser processadas, transformadas em ação e armazenadas na memória de longo prazo ou esquecidas. O esboço visuoespacial é formado por um componente espacial, visual e cinestésico, além de mecanismos de decodificação de imagens. Sua função é armazenar informações por curto prazo, como também produzir e manipular imagens mentais (Baddeley, 2000, 2009a).

Esse modelo atual da MT é uma elaboração do modelo original de três sistemas, que passaram por duas melhorias importantes. A inclusão do retentor episódico, que explica a interface entre vários domínios, tais como visuais, verbais, perceptuais e a memória de longo prazo - semântica e episódica. Esse subsistema difere da memória episódica, pela capacidade limitada (por apenas segundos) de manter informações, mas está diretamente relacionado a ela. A segunda melhoria refere-se à conexão entre a alça fonológica e o esboço visuoespacial com as memórias de longo prazo (Baddeley, 2009a).

No que se refere ao treinamento cognitivo (TC), o mesmo pode ser compreendido como um processo ativo de educação e capacitação, focado no manejo apropriado de déficits adquiridos. Como uma modalidade da (re)abilitação neuropsicológica, seu objetivo é obter o melhor potencial físico, mental e social do indivíduo, para que ele possa remanescer ou integrar-se em um meio social (Kesselring \& Beer, 2005). Em um contexto mais amplo, a reabilitação neuropsicológica visa, então, aprimorar déficits cognitivos, comportamentais e psicossociais, sendo que o TC procura otimizar habilidades cognitivas não necessariamente deficitárias (Wilson, 2008).

No envelhecimento normal, mudanças mnemônicas ocorrem de modo dissociado, sendo que alguns sistemas mnemônicos podem manter-se preservados e, até mesmo, melhorarem, enquanto outros podem sofrer declínios. No que se refere, à MT, idosos parecem ter pouca dificuldade em armazenar informações por apenas um curto período de tempo. No entanto, idosos apresentam dificuldades em realizar duplas ou múltiplas tarefas (Baddeley, 2009b; Glisky \& Glisky, 2008). De acordo com os subsistemas da MT, o avanço da idade tem certo efeito sobre a alça fonológica e o esboço visuo-espacial (Beigneux, Plaie, \& Isingrini, 2007). Entretanto, segundo Baddeley (1986), o sistema executivo central tende a ser afetado de forma mais significativa, devido a uma falha na eficiência dos sistemas frontais do cérebro durante o envelhecimento. Recentemente, também têm sido evidenciados comprometimentos relacionados ao subsistema retentor episódico (Piolino et al., 2010).

Além disso, o desempenho em várias outras funções cognitivas depende da MT e no contexto de TC essas funções dependem da estimulação da MT para serem me- lhoradas (Malouin, Belleville, Richards, Desrosiers, \& Doyon, 2004). Dessa forma, o declínio dessa função pode prejudicar o desempenho de tarefas cotidianas e a qualidade de vida dos idosos e seus familiares (Dahlin, Bäckman, Neely, \& Nyberg, 2009). Assim, observa-se que a estimulação e a preservação desse domínio cognitivo são fundamentais para esses indivíduos (Vance et al., 2008).

Embora a literatura sobre MT esteja em crescente desenvolvimento desde a década de 1970, ainda há poucos estudos sobre intervenções com tarefas específicas, não computadorizadas, para a MT, com o objetivo de aprimorar e prevenir o declínio desta função em idosos saudáveis (Duval, Coyette, \& Seron, 2008). De acordo com uma busca na literatura internacional, programas de Treinamento da Memória de Trabalho (TMT), focados em tarefas específicas para trabalhar e aprimorar esta função, não computadorizados (Duval et al., 2008; Serino et al., 2007; Vallat et al., 2005) e computadorizados (Lundqvist, Grundström, Samuelsson, \& Rönnberg, 2010; Westerberg et al., 2007), observa-se que geralmente os estudos têm sido direcionados a pessoas com prejuízos decorrentes de lesões cerebrais. No que se refere a idosos sem comprometimentos cognitivos, programas não computadorizados (Borella, Carretti, Riboldi, \& De Beni, 2010; Li et al., 2008) e computadorizados (Buschkuehl et al., 2008) parecem ser menos freqüentes até mesmo na literatura internacional. Outros estudos com esse perfil de idosos, que têm verificado o efeito de programas de treinamentos cognitivos na MT, também têm sido realizados. No entanto, estes programas de treinamentos, têm focado na estimulação de várias funções cognitivas e não têm sido específicos apenas para a MT. Dentre eles, a literatura tem apresentado aqueles não compudadorizados (Buiza et al., 2008; Craik et al., 2007; Schmiedek, Lovden, \& Lindenberger, 2010) e os computadorizados (Dahlin, Nyberg, Backman, \& Neely, 2008).

No Brasil, foi encontrada apenas uma revisão sistemática de programas de treinamento da MT para adultos e idosos, com e sem comprometimento cognitivo (Netto et al., 2010). No entanto, estudos de treinamentos da memória para idosos, sem comprometimentos cognitivos, foram encontrados para aprimorar a memória episódica (Carvalho, Neri, \& Yassuda, 2010; Yassuda, Batistoni, Fortes, \& Neri, 2006). Em âmbito nacional, até onde se sabe, nenhum estudo parece ter sido realizado ainda, para verificar o efeito de um programa de TMT, empregando tarefas específicas para aprimorar essa função em idosos saudáveis. Por outro lado, observa-se um crescente aumento de trabalhos que visam à reabilitação neuropsicológica em adultos idosos com comprometimentos cognitivos, visto que a reabilitação busca abranger serviços de orientações sistemáticas e funcionais. Neste processo, a reabilitação, procura também promover a recuperação do funcionamento cognitivo e reduzir incapacidades do individuo, auxiliando-o a reintegrar-se no meio social. Dentre as abordagens de reabilitações, treinamentos cognitivos podem estimular novas aprendizagens, 
fortalecer ou restabelecer padrões comportamentais prévios (Cicerone et al., 2000).

Diante da escassez de estudos nesta área no contexto nacional, a presente pesquisa teve como objetivo examinar o efeito de um programa de TMT no processamento da MT e de outras funções cognitivas a ela relacionadas em idosos saudáveis, procurando responder às seguintes questões específicas: (a) O TMT pode melhorar o processamento da MT? (b) A estimulação por meio de socialização pode melhorar o processamento da MT? (c) Há efeito de transferência para outras funções cognitivas após o TMT e, caso haja, para quais domínios? (d) Em caso de aprimoramento pós-intervenção, há diferenças quanto à melhora do processamento da $\mathrm{MT}$ e de outras funções cognitivas entre os grupos: experimental (TMT) e controle (Socialização)? De acordo com essas perguntas apresentadas foram formuladas as seguintes hipóteses: o TMT pode aprimorar o processamento da MT, assim como de outras funções cognitivas a ela relacionadas, tais como, memória episódica, atenção e componentes das funções executivas. Além disso, a estimulação específica através do TMT deve promover uma melhora, mais significativa e generalizada do que a socialização.

\section{Método}

\section{Participantes}

Os idosos foram recrutados no Centro Médico do Hospital Adventista Silvestre, Unidade Integrada de Prevenção, na cidade do Rio de Janeiro, especializada em pessoas com idades avançadas. Todos os participantes foram confirmados por avaliações geriátricas clínicas como idosos saudáveis e independentes. Os critérios de inclusão consistiram em: idade entre 60 e 80 anos; escolaridade de no mínimo 5 anos completos de educação formal; ausência de sintomas sugestivos de depressão, com escore máximo de 7 pontos na Escala de Depressão Geriátrica-15 itens (GDS-15), versão adaptada à popula- ção brasileira por O. P. Almeida e S. A. Almeida (1999); ausência de sintomas sugestivos de demência com pontuação $\geq 24$ pontos no Mini Exame do Estado Mental (MEEM), versão adaptada para à população brasileira por O. P. Almeida (1998). Não participaram desse estudo indivíduos idosos que apresentavam auto-relato de: diagnósticos de transtornos psiquiátricos; histórico atual ou prévio de quadros neurológicos (doenças neurodegenerativas, cerebrovasculares, traumatismo crânio encefálico, dentre outros); alcoolismo e uso de drogas ilícitas ou de benzodiazepínicos. Por fim, também não participaram desse estudo indivíduos que no último ano passaram por tratamento neuropsicológico e que apresentaram comprometimentos auditivos e/ou visuais não corrigidos. Todos esses critérios foram examinados por um questionário sociocultural e de aspectos de saúde. No decorrer da intervenção, excluíram-se aqueles que faltaram mais do que duas sessões e não puderam recuperá-las individualmente.

$\mathrm{O}$ delineamento desse estudo foi quase-experimental e comparativo com avaliação pré e pós-intervenção. A amostra inicial constituiu-se de 28 adultos idosos, 14 do grupo experimental (TMT) e 14 do controle (Socialização). Os participantes foram distribuídos aleatoriamente por meio de sorteio. No decorrer das sessões, houve perda amostral por diversos fatores: faltas freqüentes sem reposição da sessão, ocasionadas por intercorrências de saúde, falecimento de familiares e colisão de horários de atividades laborais, além do não comparecimento à avaliação neuropsicológica pós-intervenção. Assim, a amostra final ficou composta por 20 idosos, sendo nove participantes do grupo controle (três homens e seis mulheres) e 11 do grupo experimental (três homens e oito mulheres), todos brasileiros, com português como língua materna. Quanto ao sexo, não houve diferença estatística, em relação à distribuição dos participantes entre grupos, conforme o teste Qui-quadrado $(p=1,00)$. A seguir a Tabela 1 apresenta os dados sócio-demográficos e clínicos de ambos os grupos.

Tabela 1

Média e Desvio Padrão das Variáveis Socio-Demográficas e Clínicas dos Grupos

\begin{tabular}{lrrrrr}
\hline \multirow{2}{*}{ Variáveis } & \multicolumn{2}{c}{ Grupo controle } & \multicolumn{2}{c}{ Grupo experimental } \\
\cline { 2 - 6 } & \multicolumn{1}{c}{$M$} & $D P$ & $M$ & $D P$ & $p$ \\
\hline Idade & 73,00 & 4,47 & 71,55 & 5,48 & 0,530 \\
Anos de estudo & 13,89 & 2,66 & 15,09 & 3,93 & 0,446 \\
Escore no Mini Exame do Estado Mental & 28,11 & 1,76 & 27,45 & 1,50 & 0,381 \\
Escore na GDS-15' $^{1}$ & 2,33 & 2,12 & 3,00 & 1,73 & 0,449 \\
Escore da Classe Sócio-econômica $^{2}$ & 3,89 & 0,92 & 3,09 & 0,94 & 0,074 \\
Questionário Cage $^{3}$ - Escore total & 0,00 & 0,00 & 0,00 & 0,00 & - \\
Frequência de hábitos de leitura e de escrita - Escore total $^{2}$ & 12,56 & 4,69 & 16,18 & 3,34 & 0,059 \\
WAIS-III $^{4}$ Vocabulário - Total bruto & 44,44 & 6,67 & 45,73 & 8,02 & 0,706 \\
WAIS-III $^{4}$ Cubos - Total bruto & 25,56 & 9,02 & 28,45 & 10,96 & 0,533 \\
\hline
\end{tabular}

Nota. ${ }^{1}$ GDS-15 = Escala de Depressão Geriátrica; ${ }^{2}$ De acordo com o Critério de Classificação Econômica Brasil da Associação Brasileira de Empresas de Pesquisa (ABEP) de 2008; ${ }^{3}$ Histórico prévio ou atual de consumo de álcool; ${ }^{4}$ WAIS III = Escalas de Inteligência Wechsler para Adultos III. 
De acordo com a Tabela 1, pode-se observar que os grupos, experimental e controle, na linha de base, não se diferenciaram em nenhuma das variáveis sócio-demográficas (escolaridade, idade e frequência de hábitos de leitura e escrita) e clínicas (escore no Mini Exame do Estado Mental - MEEM) e na Escala de Depressão Geriátrica de 15 itens (GDS-15), e escores brutos dos subtestes $\mathrm{Cu}$ bos e Vocabulário na Escala Wechsler de Inteligência para adultos - WAIS-III. Esses resultados indicam que esses grupos comparativos, após sua distribuição randomizada, encontravam-se adequadamente emparelhados.

De acordo com os aspectos éticos, a participação dos indivíduos nesse estudo foi de forma voluntária não remunerada, tendo os mesmos assinado um Termo de Consentimento Livre e Esclarecido. O projeto desta pesquisa foi aprovado pelo Comitê de Ética em Pesquisa da Pontifícia Universidade Católica do Rio de Janeiro, sob o protocolo de número 02/2008.

\section{Procedimentos e Instrumentos}

Esta pesquisa foi conduzida em três etapas: (a) avaliação neuropsicológica pré-intervenção, (b) intervenção (TMT ou socialização), e (c) avaliação neuropsicológica pós-intervenção. Os testes neuropsicológicos pré e pósintervenção foram administrados em três sessões, com duração aproximada de uma hora e meia. No pré-teste, os procedimentos consistiram em uma entrevista de préseleção seguida das avaliações neuropsicológicas.

Após o término da avaliação pré-intervenção, o grupo experimental participou de um treinamento cognitivo da memória de trabalho (TMT), enquanto o grupo controle teve encontros de convivência social ou socialização (S). Os pré e pós-testes foram aplicados simultaneamente nos grupos experimental e controle. Ambos os programas de intervenção foram ministrados por um neuropsicólogo e um co-terapeuta, sendo que as avaliações pré e pós-intervenção foram administradas e interpretadas por neuropsicólogos cegos à intervenção. Logo após o término do período de intervenção, as avaliações neuropsicológicas aplicadas na primeira fase desse estudo (pré-teste) foram repetidas (pós-teste).

\section{Avaliação Neuropsicológica Pré e Pós-Intervenção}

Os instrumentos neuropsicológicos foram organizados em três sessões e descritos seguindo a sequência na qual foram administrados. Essa seqüência foi planejada com o objetivo de minimizar possíveis efeitos de interferência entre testes. Assim, instrumentos com tarefas predominantemente não-verbais foram intercalados com verbais. $\mathrm{Na}$ primeira sessão, além dos instrumentos de caracterização da amostra e de verificação dos critérios de inclusão, foram administrados os subtestes que examinam atenção concentrada auditiva, memória de trabalho, prospectiva, episódica verbal, e componentes das funções executivas (planejamento, iniciação e inibição verbais) do Instrumento de Avaliação Neuropsicológica Breve NEUPSILIN (Fonseca, Salles, \& Parente, 2009). Os demais subtestes que avaliam orientação temporoespacial, percepção, habilidades aritméticas, linguagem e praxias não foram administrados por tais processos não serem foco da presente investigação.

Na segunda sessão, os grupos foram examinados com os instrumentos: (a) Teste de Evocação de Buschke (Buschke \& Fuld, 1974), com evocação livre e pistas, que verificou a memória episódica visuo-verbal; (b) Teste Wisconsin de Classificação de Cartas (Heaton, Chelune, Talley, Kay, \& Curtiss, 1993), adaptado e normatizado no Brasil por Cunha et al., 2005), que investigou componentes das funções executivas, tais como, flexibilidade cognitiva, resolução de problemas, busca de estratégias, planejamento, organização e categorização; (c) Teste de Evocação de Buschke, novamente, para finalizar a evocação tardia; (d) Teste Hayling (Burgess \& Shallice, 1996, adaptado ao Português Brasileiro por Fonseca et al., 2010) que verificou funções executivas, tais como iniciação (parte A) e inibição (parte B) verbais e velocidade de processamento; (e) Teste Stroop de Cores e Palavras (Golden \& Freshwater, 2002) que mensurou atenção concentrada, inibição, velocidade de processamento e flexibilidade cognitiva, e o fenômeno de interferência do processo automático de leitura de palavras sobre a nomeação de cores; (f) Subteste Aritmética das Escalas Wechsler de Inteligência para Adultos (WAIS-III; Wechsler, 1997 adaptada para o Brasil por Nascimento, 2004). Esse subteste avaliou além das habilidades aritméticas, a velocidade de processamento e o executivo central da MT; (g) Span de Dígitos (Wechsler, 2002, adaptado para o Português Brasileiro por Fonseca et al. (2013), que verificou a atenção concentrada, memória de curto prazo (ordem direta) e também a MT (ordem indireta); (h) Subteste Semelhanças da WAIS-III, que mensurou a capacidade de estabelecer relações lógicas entre palavras, envolvendo a memória semântica.

A terceira sessão consistiu do (a) Subteste de Sequência de Números e Letras da WAIS-III, que examinou a MT; (b) Teste de Aprendizagem Auditivo-verbal de Rey (etapas 1 a 6) (Rey, 1958 adaptado por Malloy-Diniz, Lasmar, Gazinelli, Fuentes, \& Salgado, 2007), que avaliou a memória episódica imediata e tardia e habilidade de aprendizagem; (c) Subteste Cubos da WAIS-III, que mensurou a velocidade de processamento, organização e percepção visuoespaciais, e resolução de problemas lógico-abstratos; (d) Trail Making Test (Reitan, \& Wolfson, 1993, adaptado no Brasil por Fonseca et al. (2013), que investigou praxia ideomotora, atenção concentrada e alternada, flexibilidade cognitiva, planejamento visuoconstrutivo e velocidade de processamento; (e) Teste de Aprendizagem Auditivo-verbal de Rey (etapa 7 e reconhecimento), para mensurar resistência à interferência e memória episódica verbal por reconhecimento; (f) Subtestes de Fluência Verbal da Bateria Montreal de Avaliação da Comunicação - Bateria MAC (Joanette, Ska, \& Côté, 2004, adaptado por Fonseca, Parente, Côté, Ska, \& Joanette, 2008), que avaliaram funções linguísticas, mnemônicas e exe- 
cutivas (planejamento, iniciação e inibição verbais); (g) Subteste Discurso Narrativo da Bateria MAC, que avaliou a memória verbal episódica, compreensão da linguagem e processamento de inferências; (h) Subteste Vocabulário da WAIS-III, que verificou memória semântica e processamento linguístico léxico-semântico.

\section{Programa de Intervenção Treinamento da Memória de} Trabalho (TMT)

O programa de treinamento da MT (TMT), desenvolvido nesse estudo, foi realizado com o objetivo de aprimorar a MT e funções cognitivas a ela relacionadas em idosos saudáveis e independentes. Esse programa teve como base o modelo teórico da MT de Baddeley (2000) e Baddeley e Hitch (1974), e as tarefas selecionadas foram derivadas das experiências clínicas e experimentais dos autores, de estudos de estimulação da MT encontrados na literatura e das sugestões de três experts em treinamento neuropsicológico. O grau de dificuldade das tarefas no TMT foi aumentado na medida em que tarefas iniciais eram vencidas e assim outras mais difíceis eram empregadas. Esse programa foi promovido em 12 sessões semanais, com duração de uma hora e meia, por três meses e ministrado sob modalidade grupal. Na Tabela 2, o programa TMT é apresentado de modo sucinto, com os componentes da MT predominantemente estimulados e com a descrição das tarefas empregadas em cada sessão.

\section{Tabela 2}

Descrição das 12 Sessões do TMT

$\mathrm{N}^{\circ}$ Componentes MT Descrição

1

2 Esboço visuoespacial e alça fonolórica

3 Esboço visuoespacial e alça fonolórica

Retentor episódico

$6 \quad$ Executivo Central
Abertura do Grupo TMT - apresentação dos coordenadores e dos membros do grupo a partir de uma dinâmica de nomes e hobbies dos envolvidos. Psicoeducação assuntos abordados: objetivo do treinamento; definição sobre memória e aprendizagem; apresentação sobre principais teorias, tipos e queixas mnemônicas, esquecimento normal e patológico, envelhecimento, conceito e funcionamento do treinamento cognitivo.

Psicoeducação - apresentação do modelo da MT e explicação de como cada componente seria estimulado. Tarefas abordadas: Lista de palavras - Organização e escrita de uma lista de palavras a partir de uma categoria, com solicitação de evocação. Sequência de figuras - Apresentação, nomeação e evocação de cartões com imagens de frutas. Leitura - Leitura de um conto em partes. Logo após, os participantes foram solicitados a recontar cada parte, repetindo-a num segundo momento, sem apoio do coordenador do grupo.

Psicoeducação - Noção de reserva cognitiva. Tarefas abordadas: Lista de palavras e alça fonológica- Idem à sessão 2 , mas com diferentes categorias. Sequência de figuras - Idem à sessão 2, mas com figuras de redes semânticas distintas. Manipulação de imagens com cubos - adaptada de Shepard e Feng (1972), manipulação mental de imagens. Tarefa de contagem de janelas - adaptada de Baddeley (2009a). Contagem mental do número de janelas existentes na casa do melhor amigo. Figuras complexas - Apresentação de figuras complexas, por alguns segundos, através de slides. Logo após, os participantes foram solicitados a desenhá-las sem revê-las. Leitura - Idem à sessão 2, mas com um conto diferente.

Leitura - Idem à sessão 2, mas utilizando um texto de maior extensão e complexidade. Figuras complexas - Idem à sessão 3, mas com o emprego de figuras diferentes.

Vídeos Intercalados - Apresentação de dois vídeos de forma intercalada e divididos em partes semelhantes. A primeira parte do vídeo 1 foi apresentada e a seguir o participante recontou a história 1; logo após o vídeo 2 foi apresentado e outro participante recontou a história 2 , e assim por diante. No final dos dois vídeos cadaparticipante foi solicitado a contar uma parte da história, dando continuidade ao que o último tinha acabado de contar, sem apoio visual e/ou auditivo. Por fim, ambas histórias foram contadas por completo. Pistas foram dadas durante essa tarefa.

Tarefa de Figuras - adaptada de Brandimonte et al. (1992) - Figuras inteiras foram apresentadas depois retiradas, logo após os participantes foram solicitados a imaginar 
7 Executivo Central

8 Executivo central

$9 \quad$ Executivo central

10 Executivo central

11 Executivo Central e Retentor episódico

12 Todos os componentes tais figuras sem determinadas partes e dizer em que objeto se transformaram. Tarefa da Matriz - Adaptada de Toms, Morris e Foley (1994), sendo apresentada uma matriz composta por dezesseis quadrados e uma estrela num determinado quadrado. A partir dessa matriz, uma série de instruções foram passadas, quanto ao posicionamento de números nessa matriz, esse processamento foi efetuado de olhos fechados. Após essa etapa, os participantes escreveram os números de acordo com a sua memória num papel com a figura da matriz e compararam seus resultados ao esperado. Lista de palavras e aritmética - adaptada de Rosen e Engle (1997), composta pela memorização de uma lista de palavras, cada representada em um slide pela escrita e imagem, nesse mesmo slide também continha um cálculo aritmético simples. $O$ cálculo tinha que ser resolvido mentalmente e a palavra lida e memorizada. A lista foi retomada pela coordenadora do grupo após a apresentação de todos os slides. Leitura de piada - Leitura e evocação de uma piada em partes e depois por completo.

Manipulação Mental de Letras - baseada no experimento de Finke e Slayton (1988), manipulação mental das letras J e D de forma que os participantes formassem uma imagem de guarda-chuva. Lista de palavras e aritmética - Idem sessão 6, com cálculos e imagens diferentes. Tarefa da Matriz com Letras - Idem sessão 6, mas ao invés de usar números foram utilizadas letras. Leitura de piada - Idem sessão 6 .

Falta Um - Memorização de nove figuras apresentadas em um slide. Logo após, o mesmo slide foi reapresentado, faltando uma figura. Todas as figuras permaneceram no mesmo lugar, inclusive a figura ausente. Os participantes foram solicitados a dizer qual figura estava faltando. N-back - adaptado de Dobbs e Rule (1989) - Evocação de três sequências de números. Na primeira sequência, os participantes tinham que começar a evocar e anotar os números num papel a partir do segundo número ditado pelo coordenador do grupo, na segunda sequência a partir do terceiro número e na terceira sequência a partir do quarto número. $\mathrm{O} n$ ajustava o grau de dificuldade da tarefa. Vídeo - apresentação de um vídeo, dividido em partes, sobre determinada reportagem. Reconto do vídeo foi realizado primeiro em partes e depois por completo. Houve identificação de palavras-chave.

Falta Um - Idem sessão 8, mas com maior nível de dificuldade, sendo que dessa vez todas as figuras trocam de posições. Meses - adaptada de Serino et al. (2007). Apresentação de quatro nomes de meses em diferentes sequências do calendário. Os p a rticipantes tiveram que ordená-los mentalmente de acordo com a sequência do calendário e responder qual o mês que vinha primeiro. Números e Letras - adaptado de Vallat et al. (2005). Apresentação verbal de palavras e a contagem mental do número de letras em cada palavra. Vídeo - Idem sessão 8, mas diferente vídeo foi apresentado.

Falta Um - Idem sessão 8, mas com maior nível de dificuldade, dessa vez faltaram duas figuras no slide e todas mudaram de posição. Fórmula Alfabética - adaptado de Vallat et al. (2005). Apresentação de contas matemáticas simples com números e letras. Por exemplo: A + 2 = C. Número de Letras - Idem sessão 9, mas empregando diferentes palavras. Vídeo - Idem sessão 8, mas com um vídeo diferente.

Imagens e texto - Apresentação de imagens relacionadas ao texto a ser apresentado. A seguir, leitura do texto em partes, seguidas de seus respectivos reconto e logo após identificação de palavras-chave desse texto. As imagens foram novamente apresentadas e finalmente o reconto do texto integral foi solicitado. Palavras em ordem alfabética - adaptada de Vallat et al. (2005). Palavras foram apresentadas oralmente e os participantes foram solicitados a escrevê-las em ordem alfabética de acordo com a primeira, segunda ou até a quinta letra. Por exemplo: partir/proteção/protestos. Vale ressaltar que essas palavras foram retiradas do texto previamente trabalhado.

Imagens e texto - Idem sessão 11, mas utilizando outras imagens e texto. Fechamento das 12 sessões do treinamento - Revisão e retomada das técnicas trabalhadas durante o programa, e recapitulação de como elas se aplicam ao cotidiano. 
Pode-se observar na Tabela 2 que várias sessões apresentaram técnicas de psicoeducação, para conscientizar e sensibilizar os participantes sobre a conexão entre tarefas da MT realizadas durante o TMT e seus cotidianos, motivando assim esses participantes a aderirem ao programa. Além dessas técnicas específicas, no decorrer das sessões, procurou-se estimular todos os quatros subsistemas da MT.

\section{Socialização}

A atividade de socialização realizada com o grupo controle consistiu de encontros sociais nos quais temas diversos, tais como vinhos, olimpíadas e educação, eram discutidos e inicialmente apresentados pelo coordenador do grupo. Esses temas motivavam um debate de forma descontraída e informal entre os participantes que mudava no decorrer dos encontros. $\mathrm{O}$ grupo desconhecia os temas para evitar um preparo prévio e transformar os encontros de socialização em treinamento cognitivo. Esse grupo se reuniu uma vez por semana, por uma hora e meia, durante 12 semanas, tendo como líder um neuropsicólogo.

\section{Análise de Dados}

A partir do Teste Kolmogorov-Smirnov, realizado no programa SPSS versão 16.0, observou-se que os dados comportaram-se de forma não paramétrica. Em função disso, foram feitas análises estatísticas descritivas (medianas e amplitudes) e comparou-se o desempenho nos testes neuropsicológicos intra-grupos pelo teste Wilcoxon, e entre-grupos no pré e pós-teste pelo Mann-Whitney, com $p \leq 0,05$.

\section{Resultados}

A mediana e a amplitude do desempenho nas tarefas da avaliação neuropsicológica pré e pós-intervenção dos grupos, experimental e controles, estão apresentadas na Tabela 3. Além disso, também estão expostos os valores de $p$ derivados da análise comparativa do teste Wilcoxon intra-grupos dos instrumentos neuropsicológicos.

Os resultados da Tabela 3 indicam que o grupo experimental apresentou diferenças significativas entre pré e pós-intervenção em tarefas que exigiam atenção concentrada, memória de curto prazo, aprendizagem e memória episódica, com um desempenho neuropsicológico superior no período pós-intervenção. Ressalta-se que na avaliação pós-teste houve uma redução do escore na evocação de palavras com pistas no Teste Buschke. Isso ocorreu porque o grupo experimental precisou de menos pistas para auxiliar no processo de evocação mnemônica, melhorando, assim, o escore de evocação livre. O grupo controle também se diferenciou em tarefas que avaliaram atenção concentrada e memória episódica, demonstrando uma melhora no desempenho após encontros de socialização. No entanto, observa-se que o grupo experimental obteve melhora em um número superior de tarefas (nove escores de desempenho) quando comparado ao grupo controle (três escores).

Em complementaridade, a análise comparativa intragrupos, constatou-se também diferenças significativas entre os grupos na avaliação pré-intervenção no escore da tarefa NEUPSILIN, Memória de Trabalho Ordenamento de Dígitos - Maior Sequência Repetida ( $U=18,0$; $p=0,016$ ). Além desse resultado, observou-se também diferenças significativas entre grupos na avaliação pósintervenção no número de categorias completadas no Wisconsin, $(U=24,0 ; p=0,032)$. Finalmente a análise entre grupos detectou uma diferença significativa na memória episódica avaliada através dos seguintes escores da tarefa do Rey Verbal: número de evocações no total de palavras do A7 ( $U=22,5 ; p=0,039)$, escore total da tarefa de reconhecimento de palavras da lista A ( $U=13,5 ; p=0,006)$, e interferência retroativa $(U=23,5$; $p=0,047)$.

\section{Discussão}

Os dados obtidos no presente estudo verificaram a ocorrência do efeito terapêutico de um programa de intervenção neuropsicológica, na modalidade de treinamento da MT, um dos domínios cognitivos mais complexos da cognição humana. De um modo geral, os grupos experimental e controle apresentaram melhoras cognitivas em diferentes níveis.

No que diz respeito às duas primeiras questões de pesquisa “(a) $\mathrm{O}$ treinamento da MT pode melhorar o processamento desta função?” e “(b) A estimulação por meio da socialização pode melhorar o processamento da MT?”, formularam a hipótese de que o TMT pode aprimorar o processamento da MT foi apenas parcialmente confirmada, pois os dados comparativos pré e pós-intervenção do grupo experimental sugeriram que componentes da alça fonológica e retentor episódico apresentaram melhora, o que não foi observado nas tarefas de mensuração do executivo central. No que se refere à segunda hipótese formulada de que a socialização também pode promover uma melhora, entretanto menos significativa e generalizada do que o grupo experimental foi confirmada, pois diferenças significativas foram apresentadas em menor número de variáveis no grupo de socialização (4 variáveis) do que no grupo experimental (10 variáveis).

A hipótese gerada pela terceira questão, “(c) Haverá efeito de transferência para outras funções cognitivas após o TMT e, caso ocorra, para quais domínios?", de que o TMT pode aprimorar o processamento de outras funções cognitivas relacionadas à $\mathrm{MT}$, tais como, memória episódica, atenção e componentes da função executiva, foi confirmada pelos resultados intra-grupos, através da melhora da memória episódica visuo-verbal, auditivoverbal e da atenção e, dos resultados entre grupos através da melhora das funções executivas e da memória episódica verbal. Esses resultados mostraram que o presente estudo mesmo com uma amostra reduzida, ainda relatou 
Psicologia: Reflexão e Crítica, 26(1), 122-135.

$\frac{0}{0} e_{0}^{0}$

.

0
0
0
0
0
$\circ$
0
0

竞

常

号

劳

苛

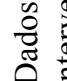

要

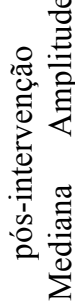

票
告

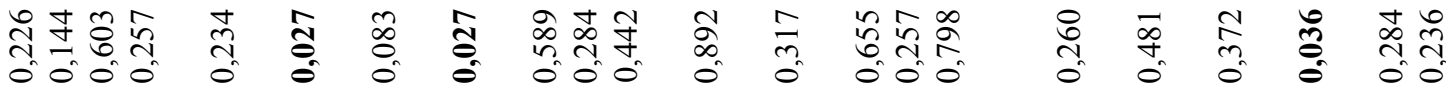

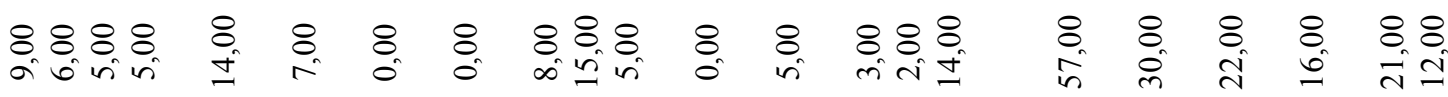

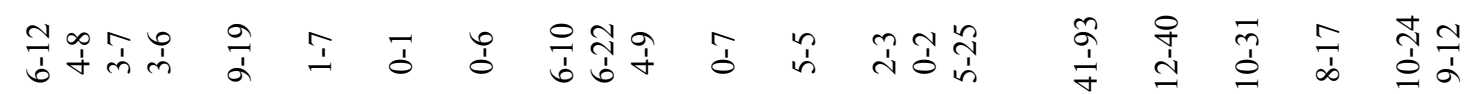

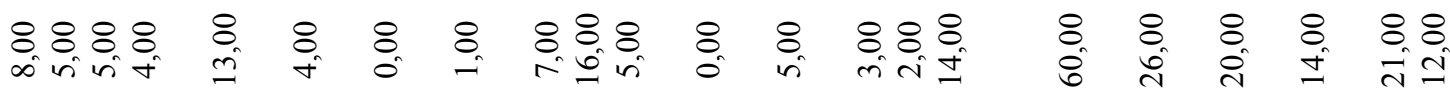

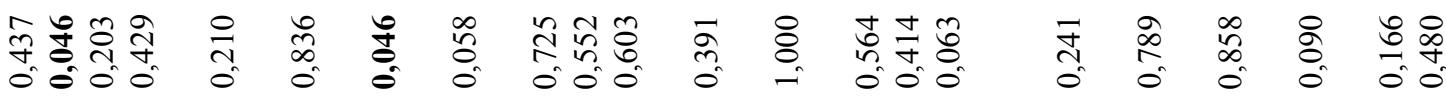

2

:

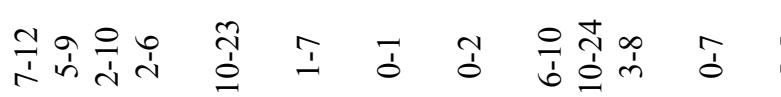

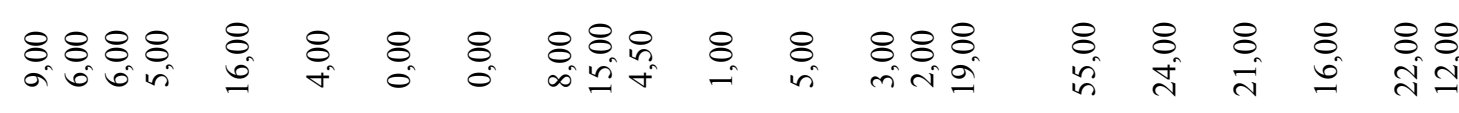

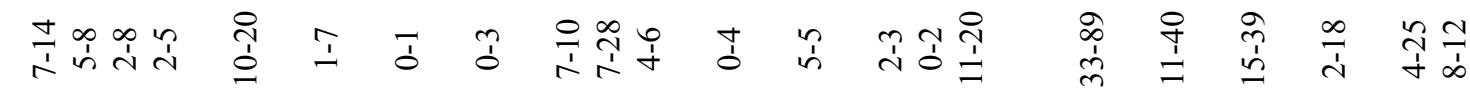

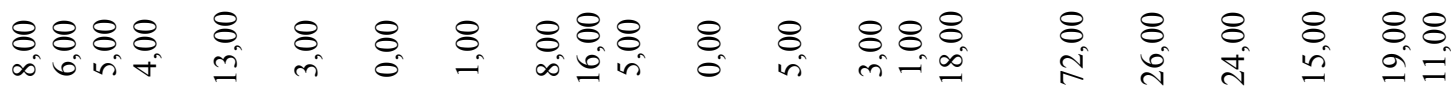

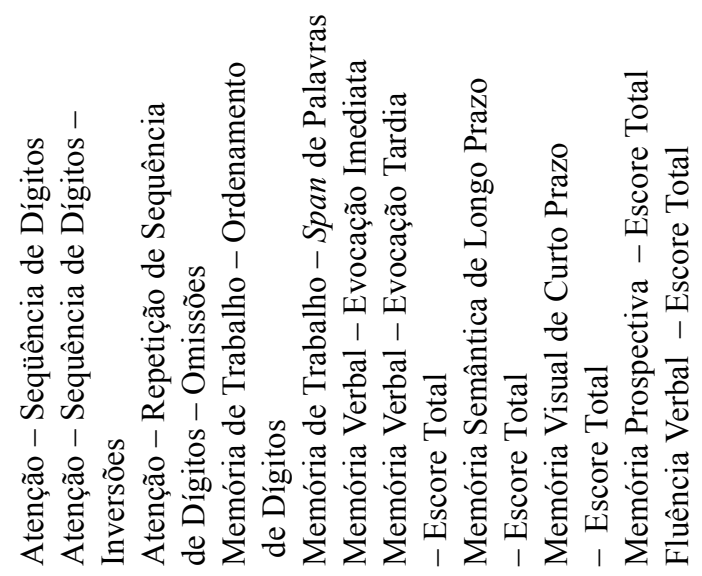

NITISdกAN

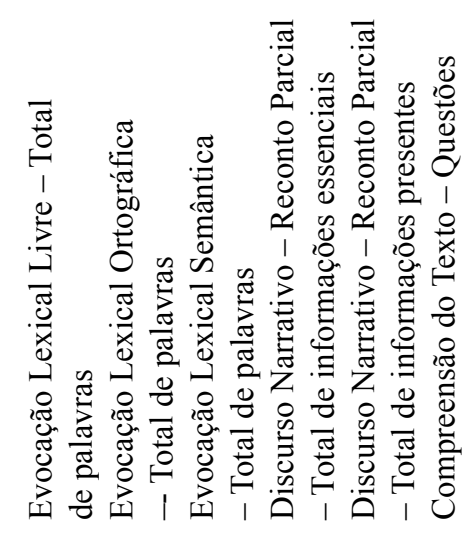

गVW VIУALLG 
Netto, T. M., Greca, D. V., Zimmermann, N., Oliveira, C. R., Teixeira-Leite, H. M., Fonseca, R. P. \& Landeira-Fernandez, J. (2013). Efeito de um Programa de Treinamento da Memória de Trabalho em Adultos Idosos.

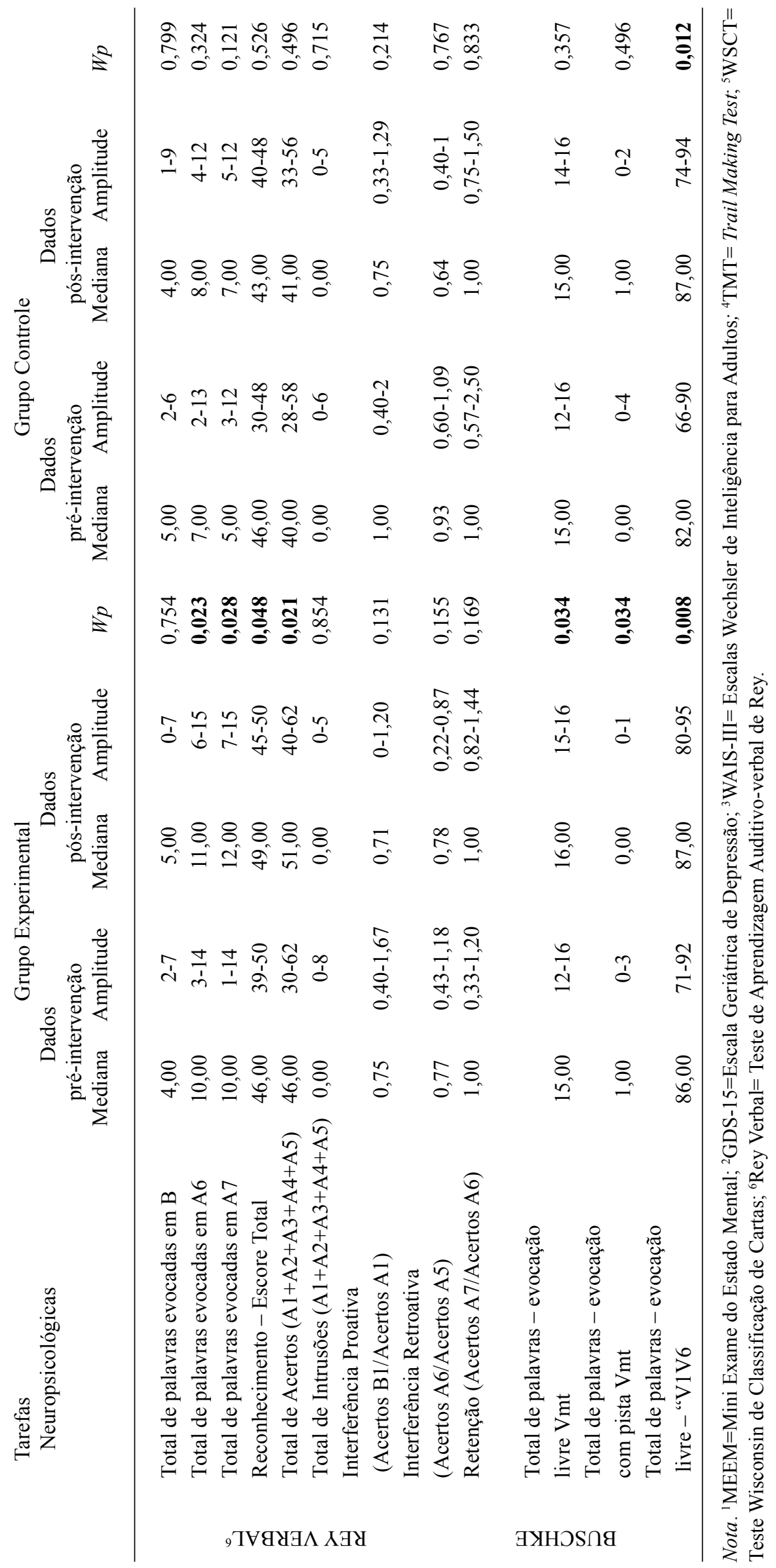


uma diferença significativa no que se refere ao efeito de transferência, contradizendo assim, Dahlin et al. (2009), que apontam o fenômeno de transferência como sendo pouco evidente em estudos de intervenções. Tal fenômeno ocorreu com menor intensidade para a atenção do que para a memória episódica. Esse sistema mnemônico explícito está fortemente relacionado ao processamento dos componentes alça fonológica, esboço visuoespacial e retentor episódico da MT (Baddeley, 2000; Baddeley \& Hitch, 1974). Outra hipótese explicativa, embasada no modelo da relação entre componentes cognitivos, abordado por Salthouse (1994; Salthouse \& Babcock, 1991), em seus estudos sobre envelhecimento cognitivo, defende a associação entre MT, velocidade de processamento, capacidade de armazenamento e inteligência fluida (Salthouse, 1994; Salthouse \& Babcock, 1991; Salthouse \& Pik, 2008). Devido à relação entre estes componentes e a MT, o treinamento desta última, pode melhorar indiretamente os demais processamentos associados.

Quanto à última questão de pesquisa, "(d) Em caso de aprimoramento pós-intervenção, haverá diferenças quanto à melhora do processamento da MT e de outras funções cognitivas entre um grupo estimulado pelo TMT e outro pela socialização?", a hipótese formulada de que a estimulação específica da MT através do TMT deveria promover melhora mais significativa e generalizada do que a socialização foi confirmada. Em geral, o grupo experimental teve uma melhora significativa no desempenho em tarefas de atenção, memória de curto prazo, aprendizagem e memória episódica. No entanto, o grupo controle teve uma melhora significativa em um menor número de testes na atenção e na memória episódica. O efeito observado no grupo submetido à socialização pode ter ocorrido por uma estimulação cognitiva indireta, devido à exposição a um contexto social estruturado e também devido a maior motivação após convivência com pares (Depp, Vahia, \& Jeste, 2010; Reichstadt, Depp, Palinkas, Folsom, \& Jeste, 2007). Embora a socialização tenha tido um efeito menor do que o TMT, o grupo controle teve melhoras em algumas tarefas em que o grupo experimental não teve como no subteste do NEUPSILIN da atenção - Sequência de dígitos e Repetição de seqüência de dígitos - omissões e no subteste da bateria MAC Discurso Narrativo Reconto Parcial - Informações Essenciais, sugerindo assim que há características do grupo controle que podem beneficiar o grupo experimental, por exemplo, discussão de assuntos pertinentes à realidade diária dos membros do grupo, que pode aprimorar a atenção, a memorização e síntese de informações essenciais.

$\mathrm{Na}$ tentativa de melhor compreender as melhoras observadas, mesmo que assimétricas, no grupo diretamente estimulado pelo TMT e pela socialização, um terceiro grupo sem nenhum tipo de intervenção poderia ter sido incluído para proporcionar um delineamento comparativo mais sólido, para testar de forma mais eficiente evidências de efeito terapêutico do TMT (Cicerone et al.,
2000). Convergentemente, no estudo de Buiza et al. (2008) três grupos foram incluídos. O primeiro grupo experimental obteve treino cognitivo junto a atividades de socialização; o segundo também experimental realizou somente treino cognitivo e o grupo controle não vivenciou nenhum tipo de intervenção. De acordo com esse estudo o grupo mais beneficiado foi o que participou do treinamento cognitivo, junto com outras atividades que estimularam diferentes aspectos da vida desses indivíduos, indicando, assim, que o treinamento de funções cognitivas associado a outras atividades relacionadas à socialização, pode superar os resultados de um treino cognitivo per si.

Os resultados da presente pesquisa estão de acordo com a literatura atual sobre estudos que avaliaram a efetividade de TMT em adultos idosos saudáveis e independentes. Dentre esses estudos, um deles que empregou treinamento específico da MT, relatou diferenças significativas somente no aprimoramento do subsistema esboço visuoespacial da MT e não nos outros subsistemas como, alça fonológica, retentor episódico e executivo central (Buschkuehl et al., 2008). Adicionalmente, outro estudo, que utilizou treinamento global, incluindo funções cognitivas além da MT, não relatou diferenças na melhora dos subsistemas da MT (Craik et al., 2007). Ambas as investigações tiveram amostras pequenas e intervenções de curta duração (apenas três meses). Um terceiro estudo, que investigou o efeito de um programa de treinamento cognitivo global com uma amostra grande $(n=238)$ e duração mais prolongada, de dois anos, foi o único que mostrou melhora na MT. Tal melhora foi relatada apenas no segundo ano de treinamento cognitivo (Buiza et al., 2008).

De acordo com a literatura, foram encontrados apenas outros cinco estudos que investigaram o efeito de intervenções de TMT na MT e em outras habilidades cognitivas a ela relacionadas. Entretanto, esses estudos investigaram amostras pequenas e clínicas com lesões cerebrais focais. Todos empregaram programas específicos de aprimoramento da MT e apresentaram resultados positivos em relação à melhora dessa função cognitiva, principalmente em relação ao sistema executivo central (Duval et al., 2008; Serino et al., 2007; Vallat et al., 2005; Vallat-Azouvi, Pradat-Diehl, \& Azouvi, 2009; Westerberg et al., 2007). Tal aprimoramento tende a ser mais evidente em indivíduos após lesões cerebrais, na medida em que, na linha de base, os escores de desempenho nas tarefas neuropsicológicas desses indivíduos, tenham sido provavelmente inferiores aos escores de idosos saudáveis (Borella et al., 2010; Engvig et al., 2010; Netto et al., 2010).

Esses resultados positivos em relação à melhora da MT em amostras clínicas com lesões cerebrais focais sugerem que o aprimoramento da MT, talvez seja mais propenso a acontecer quando se trata de sequelas de quadros neurológicos, quando resultados nos desempenhos em testes neuropsicológicos na linha de base mostram-se mais 
distantes das normas, do que em indivíduos saudáveis. Esses resultados podem ocorrer, ainda, com maior frequência em intervenções específicas, porque as tarefas estimulam diretamente as funções cognitivas alvo. Assim, a relativa limitação do presente estudo de haver encontrado efeitos não tão robustos após o TMT pode ser entendida como um achado inerente a estudos de estimulação de participantes saudáveis, conforme mencionado por Buschkuehl et al. (2008).

Nesses programas com amostras clínicas, a duração de curto prazo (de três a seis meses) não pareceu interferir no aprimoramento da MT, como nos estudos com adultos idosos saudáveis. Segundo Cicerone et al. (2005), a efetividade das estratégias compensatórias de memória parece depender da gravidade dos déficits mnemônicos. No entanto, os estudos avaliados sobre efeito do TMT em idosos saudáveis parecem sugerir que se o programa de intervenção tiver a duração de dois anos e uma frequência de no mínimo duas vezes por semana, torna-se maior a possibilidade de obter resultados mais positivos.

Algumas limitações foram apresentadas pelo presente estudo, dentre elas destacam-se: a duração das intervenções durante um período de curto prazo, que muitas vezes é imposto pelo próprio idoso (compromissos de viagens, cirurgias, doenças e família) e/ou pela instituição (limite de horários, disponibilidade de espaço, tempo e custos); tamanho pequeno dos grupos; amostra constituída por idosos de alto desempenho; ausência de testagem ecológica para verificar funcionalidade dos participantes no cotidiano antes e após intervenção e a quantidade elevada de variáveis testadas. Além dessas limitações, outra, que também é relatada em diferentes estudos, foi o efeito de aprendizagem, que pode ocorrer entre teste e reteste. Muitas vezes, isso acontece devido à falta de versões equivalentes aos mesmos instrumentos neuropsicológicos administrados. Nesse estudo a mesma bateria foi empregada na pré e pós intervenção em espaço de tempo de apenas três meses.

Considerando-se que durante o envelhecimento normal a MT e a memória episódica estão dentre aquelas que sofrem maior declínio (Düzel et al., 2010) e que os resultados desse estudo mostraram o quanto é possível aprimorar a memória episódica, relacionada diretamente ao processamento dos subsistemas da MT, sugere-se que o TMT pode ser útil no contexto da neuropsicologia do envelhecimento. Considera-se possível então, auxiliar idosos na estimulação desses domínios cognitivos e possibilitar que profissionais na área da saúde prevejam ou contenham declínios nessas memórias, mantendo a independência funcional e qualidade de vida desses indivíduos durante o envelhecimento.

Como continuidade, torna-se importante administrar o TMT no grupo controle, e verificar o efeito de aprimoramento da MT. Faz-se também relevante a realização de estudos de acompanhamento, para averiguar a duração deste efeito em médio e longo prazo como várias pesqui- sas vem conduzindo (Borella et al., 2010; Buiza et al., 2008; Buschkuehl et al., 2008).

Pela escassez de literatura nessa área, sugere-se que outros estudos sejam desenvolvidos, comparando grupos com: (a) treino específico da MT, (b) treino global da memória, (c) socialização e (d) grupo de espera, sem nenhuma intervenção. Todos os integrantes dos grupos deverão ter características de convivência social frequente, visando assim controlar a estimulação cognitiva natural promovida pela socialização.

Por fim, na interface cada vez mais explorada entre neuropsicologia e avaliação com técnicas avançadas de neuroimagem, estudos que busquem evidências de melhora neurobiológica estrutural e/ou funcional após treinamento cognitivo podem ser complementares e importantes para a neuropsicologia cognitiva e do desenvolvimento, como o de Engvig et al. (2010).

Em suma, os resultados obtidos nesse estudo sugeriram a necessidade de programas mais frequentes e duradouros, com grupos controles que não sejam indiretamente estimulados, como no caso de socialização. Além disso, ressalta-se que as conclusões apresentadas devem ser interpretadas considerando-se o pequeno tamanho amostral do estudo. No entanto, até onde se sabe, é um dos poucos trabalhos em nível nacional que apresenta um método de intervenção de memória de trabalho utilizado num contexto brasileiro, representando um valor heurístico-metodológico. Espera-se que os presentes achados contribuam para a prática clínica e futuras investigações com amostras maiores replicando o método apresentado neste estudo.

\section{Referências}

Almeida, O. P. (1998). Mini Exame do Estado Mental e o diagnóstico de demência no Brasil. Arquivos de Neuropsiquiatria, 56(3-B), 605-612.

Almeida, O. P., \& Almeida, S. A. (1999). Confiabilidade da versão brasileira da Escala de Depressão em Geriatria (GDS) versão reduzida. Arquivos de Neuropsiquiatria, 57(2-B), 421426.

Associação Brasileira de Empresas de Pesquisa. (2008). Critério de Classificação Econômica Brasil. Retrieved from http:/ /www.abep.org/novo/FileGenerate.ashx?id=257

Baddeley, A. D. (1986). Working memory. Oxford, UK: Clarendon Press.

Baddeley, A. D. (2000). The episodic buffer: A new component of working memory? Trends in Cognitive Sciences, 4(11), 417-423.

Baddeley, A. D. (2003). Working memory and language: An overview. Journal of Communication Disorders, 36, 189-208.

Baddeley, A. D. (2009a). Working memory. In A. D. Baddeley, M. W. Eysenck, \& M. C. Anderson (Eds.), Memory (pp. 4168). New York: Psychology Press.

Baddeley, A. D. (2009b). Memory and aging. In A. D. Baddeley, M.W. Eysenck, \& M. C. Anderson (Eds.), Memory (pp. 293315). New York: Psychology Press.

Baddeley, A. D. (2010). Working memory. Current Biology, 20(4), 136-140. 
Baddeley, A. D., Allen, R., \& Vargha-Khadem, F. (2010). Is the hippocampus necessary for visual and verbal binding in working memory? Neuropsychologia, 48, 1089-1095.

Baddeley, A. D., \& Hitch, G. J. (1974). Working memory. In G. A. Bower (Ed.), Recent advances in learning and motivation (pp. 47-90). New York: Academic Press.

Beigneux, K., Plaie, T., \& Isingrini, M. (2007). Aging effect on visual and spatial components of working memory. International Journal of Aging and Human Devevelopment, 65(4), 301-314.

Borella, E., Carretti, B., Riboldi, F., \& De Beni, R. (2010). Working memory training in older adults: Evidence of transfer and maintenance effects. Psychology and Aging, 25(4), 767778.

Brandimonte, M., Hitch, G., \& Bishop, D. (1992). Verbal recording of visual stimuli impairs mental image transformations. Memory and Cognition, 20(4), 449-455.

Buiza, C., Etxeberria, I., Galdona, N., Gonzalez, M. F., Arriola, E., Lopez de Munain, A., et al. (2008). A randomized, twoyear study of the efficacy of cognitive intervention on elderly people: The Donostia Longitudinal Study. International Journal of Geriatric Psychiatry, 23, 85-94.

Burgess, P. W., \& Shallice, T. (1996). Response suppression, initiation, and strategy use following frontal lobe lesions. Neuropsychologia, 34(1), 263-273.

Buschke, H., \& Fuld, P. A. (1974). Evaluation of storage, retention, and retriving in disordered memory and learning. Neurology, 11, 1019-1025.

Buschkuehl, M., Jaeggi, S. M., Hutchison, S., Perrig-Chiello, P., Dapp, C., Muller, M., et al. (2008). Impact of working memory training on memory performance in old-old adults. Psychology and Aging, 23, 743-753.

Cabeza, R., Daselaar, S. M., Dolcos, F., Prince, S. E., Budde, M., \& Nyberg, L. (2004). Task-independent and task-specific age effects on brain activity during working memory, visual attention and episodic retrieval. Cerebral Cortex, 14, 364 375.

Carvalho, F. C., Neri, A. L., \& Yassuda, M. S. (2010). Treino de memória episódica com ênfase em categorização para idosos sem demência e depressão. Psicologia: Reflexão e Crítica, 23(2), 317-323.

Cicerone, K. D., Dahlberg, C., Kalmar, K., Langenbahn, D. M., Malec, J. F., Bergquist, T. F., et al. (2000). Evidencebased cognitive rehabilitation: Recommendations for clinical practice. Archives of Physical Medicine and Rehabiltation, 81, 1596-1615.

Cicerone, K. D., Dahlberg, C., Malec, J. F., Langenbahn, D. M., Felictti, T., Kneipp, S., et al. (2005). Evidence-based cognitive rehabilitation: Updated review of the literature from 1998 through 2002. Archives of Physical Medicine and Rehabiltation, 86, 1681-1692.

Craik, F. I. M., Winocur, G., Palmer, H., Binns, M. A., Edwards, M., Bridges, K., et al. (2007). Cognitive rehabilitation in the elderly: Effects on memory. Journal of the International Neuropsychological Society, 13, 132-142.

Cunha, J. A., Trentini, C. M., Argimon, I. L., Oliveira, M. S., Werlang, B. G., \& Prieb, R. G. (2005). Teste Wisconsin de Classificação de Cartas: Manual revisado e ampliado. São Paulo, SP: Casa do Psicólogo.

Dahlin, E., Bäckman, L., Neely, A. S., \& Nyberg, L. (2009). Training of the executive component of working memory: Subcortical areas mediate transfer effects. Restorative Neurology and Neuroscience, 27(5), 405-419.
Dahlin, E., Nyberg, L., Backman, L., \& Neely, A. S. (2008). Plasticity of executive functioning in young and older adults: Immediate training gains, transfer, and long-term maintenance. Psychology and Aging, 23(4), 720-730.

Depp, C., Vahia, I. V., \& Jeste, D. (2010). Successful aging: Focus on cognitive and emotional health. Annual Review of Clinical Psychology, 6, 527-550.

Dobbs, A., \& Rule, B. (1989). Adult age differences in working memory. Psychology and Aging, 4(4), 500-503.

Duval, J., Coyette, F., \& Seron, X. (2008). Rehabilitation of the central executive component of working memory: A reorganization approach applied to a single case. Neuropsychological Rehabilitation, 18(4), 430-460.

Düzel, S., Münte, T. F., Lindenberger, U., Bunzeck, N., Schütze, H., Heinze, H.-J., et al. (2010). Basal forebrain integrity and cognitive memory profile in healthy aging. Brain Research, 1308, 124-136.

Engvig, A., Fjell, A. M., Westlye, L. T., Moberget, T., Sundseth, Ø., Larsen, V. A., et al. (2010). Effects of memory training on cortical thickness in the elderly. Neuroimage, 52, 1667-1676.

Finke, R. A., \& Slayton, K. (1988). Explorations of creative visual synthesis in mental imagery. Memory and Cognition, 16, 252-257.

Fonseca, R. P., Parente, M. A. M. P., Côté, H., Ska, B., \& Joanette, Y. (2008). Apresentando um instrumento de avaliação da comunicação à Fonoaudiologia Brasileira: Bateria MAC. Pró-Fono Revista de Atualização Científica, 20(4), 285-292.

Fonseca, R. P., Salles, J. F., \& Parente, M. A. M. P. (2009). Instrumento de Avaliação Neuropsicológica Breve NEUPSILIN. São Paulo, SP: Vetor.

Fonseca, R. P., Oliveira, C. R., Gindri, G., Zimmermann, N., Reppold, C. T., \& Parente, M. A. M. P. (2010). Teste Hayling: Um instrumento de avaliação de componentes das funções executivas. In C. S. Hutz (Ed.), Avanços em Avaliação Psicológica e Neuropsicológica de crianças e adolescentes (pp. 337-364). São Paulo, SP: Casa do Psicólogo.

Fonseca et al. (2013). Instruments of executive functions assessment: Preliminary normative data and sociodemographic studies. Manuscrito em preparação.

Glisky, E. L., \& Glisky, M. L. (2008). Memory rehabilitation in older adults. In D. T. Stuss, G. Winocur, \& I. H. Robertson (Eds.), Cognitive neurorehabilitation: Evidence and application ( ${ }^{\text {nd }}$ ed., pp. 541-562). Cambridge, England: Cambridge University Press.

Golden, C. J., \& Freshwater, S. M. (2002). The Stroop Color and Word Test: A manual for clinical and experimental uses. Chicago, IL: Stoelting.

Heaton, R. K., Chelune, G. J., Talley, J. L., Kay, G. G., \& Curtiss, G. (1993). Wisconsin Card Sorting Test Manual. Odessa, TX: Psychological Assessessment Resources.

Joanette, Y., Ska, B., \& Côté, H. (2004). Protocole MEC Protocole Montreál d'Évaluation de la Communication. Montreal, Canada: Ortho Édition.

Kesselring, J., \& Beer, S. (2005). Symptomatic therapy and neurorehabilitation in multiple sclerosis. The Lancet Neurology, 4(10), 643-652.

Leigh, E., \& Hirsch, C. R. (2011). Worry in imagery and verbal form: Effect on residual working memory capacity. Behaviour Research and Therapy, 49(2), 99-105.

Levens, S. M., \& Gotlib, I. H. (2010). Updating positive and negative stimuli in working memory in depression. Journal of Experimental Psychology: General, 139(4), 654-664. 
Netto, T. M., Greca, D. V., Zimmermann, N., Oliveira, C. R., Teixeira-Leite, H. M., Fonseca, R. P. \& Landeira-Fernandez, J. (2013). Efeito de um Programa de Treinamento da Memória de Trabalho em Adultos Idosos.

Li, S. C., Schmiedek, F., Huxhold, O., Rocke, C., Smith, J., \& Lindenberger, U. (2008). Working memory plasticity in old age: Practice gain, transfer, and maintenance. Psychology and Aging, 23(4), 731-742.

Lundqvist, A., Grundström, K., Samuelsson, K., \& Rönnberg J. (2010). Computerized training of working memory in a group of patients suffering from acquired brain injury. Brain Injury, 24(10), 1173-1183.

Malouin, F., Belleville, S., Richards, C. L., Desrosiers, J., \& Doyon, J. (2004). Working memory and mental practice outcomes after stroke. Archives of Physical Medicine and Rehabilitation, 85, 177-183.

Malloy-Diniz, L. F., Lasmar, V. A. P., Gazinelli, L. S. R., Fuentes, D., \& Salgado, J. V. (2007). The Rey Auditory-Verbal Learning Test: Applicability for the Brazilian elderly population. Revista Brasileira de Psiquiatria, 29, 324-329.

Nascimento, E. (2004). Escala de Inteligência Wechsler para Adultos WAIS - III. São Paulo, SP: Casa do Psicólogo.

Netto, T. M., Greca, D. V., Zimmermann, N., Oliveira, C., Fonseca, R. P., \& Landeira-Fernandez, J. (2010). Working memory intervention programs for adults - A systematic review. Dementia \& Neuropsychologia, 4(3), 222-231.

Norman, D. A., \& Shallice, T. (1986). Attention to action: Willed and automatic control of behaviour. In R. J. Davidson, G. E. Schwarts, \& D. Shapiro (Eds.), Consciousness and selfregulation: Advances in research and theory (pp. 1-18). New York: Plenum Press.

Oliveira, R. M. (2007). O conceito de Executivo Central e suas origens. Psicologia: Teoria e Pesquisa, 23(4), 399-406.

Piolino, P., Coste, C., Martinelli, P., Macé, A.-L., Quinette, P., Guillery-Girard, B., et al. (2010). Reduced specificity of autobiographical memory and aging: Do the executive and feature binding functions of working memory have a role? Neuropsychologia, 48, 429-440.

Reichstadt, J., Depp, C. A., Palinkas, L. A., Folsom, D. P., \& Jeste, D. V. (2007). Building blocks of successful aging: A focus group study of older adults' perceived contributors to successful aging. The American Journal of Geriatric Psychiatry, 15(3), 194-201.

Reitan, R. M., \& Wolfson, D. (1993). The Halstead - Reitan neuropsychological test battery: Theory and clinical interpretation ( $2^{\text {nd }}$ ed.). Tucson, AZ: Neuropsychology Press.

Rey, A. (1958). L'examen Clinique en Psychologie. Paris: Presse Universitaire de France.

Rosen, V., \& Engle, R. (1997). The role of working memory capacity in retrival. Journal of Experimental Psychology: General, 126(3) 211-227.

Salthouse, T. A. (1994). The aging of working memory. Neuropsychology, 8(4), 535-545.

Salthouse, T. A., \& Babcock, R. L. (1991). Decomposing adult age differences in working memory. Developmental Psychology, 27(5), 763-776.

Salthouse, T. A., \& Pink, J. (2008). Why is working memory related to fluid intelligence? Psychonomic Bulletin \& Review, 15(2), 364-371.

Schmiedek, F., Lovden, M., \& Lindenberger, U. (2010). Hundred days of cognitive training enhance broad abilities in adulthood: Findings from the COGITO study. Frontiers in Aging Neuroscience, 2(27), 1-10.

Serino, A., Ciaramelli1, E., Santantonio, A. D., Malagù, S., Servadei, F., \& Làdavas, E. (2007). A pilot study for rehabilitation of central executive deficits after traumatic brain injury. Brain Injury, 21(1), 11-19.
Shepard, R., \& Feng, C. (1972). A chronometric study of mental paper-folding. Cognitive Psychology, 3(2), 228-243.

Toms, M., Morris, N., \& Foley, P. (1994). Characteristics of visual interference with visuospatial working memory. British Journal of Psychology, 85, 131-144.

Vallat, C., Azouvi, P., Hardisson, H., Meffert, R., Tessier, C., \& Pradat-Diehl, P. (2005). Rehabilitation of verbal working memory after left hemisphere stroke. Brain Injury, 19(13), 1157-1164.

Vallat-Azouvi, C., Pradat-Diehl, P., \& Azouvi, P. (2009). Rehabilitation of the central executive of working memory after a severe traumatic brain injury: Two single-case studies. Brain Injury, 23, 585-594.

Vance, D. E., Webb, N. M., Marceaux, J. C., Viamonte, S. M., Foote, A. W., \& Ball, K. K. (2008). Mental stimulation, neural plasticity, and aging: Directions for nursing research and practice. Journal of Neuroscience Nursing, 40(4), 241-249.

Wechsler, D. (1997). Wechsler Adult Intelligence Scale-III. San Antonio, TX: The Psychological Corporation.

Wechsler, D. (2002). Memory Scale. San Antonio, TX: Psychological Corporation.

Westerberg, H., Jacobaeus, H., Hirvikoski, T., Clevberger, P., Ostensson, M. L., Bartfai, A., et al. (2007). Computerized working memory training after stroke - A pilot study. Brain Injury, 21(1), 21-29.

Wilson, B. A. (2008). Neuropsychological rehabilitation. Annual Review of Clinical Psychology, 4, 141-162.

Yassuda, M. S., Batistoni, S. S. T., Fortes, A. G., \& Neri, A. L. (2006). Treino de memória no idoso saudável: Benefícios e mecanismos. Psicologia: Reflexão e Crítica, 19(3), 470-481.
Recebido: 14/12/2010 $1^{a}$ revisão: 30/06/2011 $2^{a}$ revisão: $17 / 11 / 2011$ Aceite final: 21/11/2011 